\title{
INVESTIGACIONES
}

\section{Cómo formar al profesorado de secundaria para satisfacer las necesidades educativas de la próxima década}

\author{
How to teach secondary school teachers \\ to meet the educational needs of the next decade
}

Carolina Falcón-Linares ${ }^{a}$

${ }^{a}$ Facultad de Educación. Universidad de Zaragoza, España. cfalcon@carolinafalcon.es

\section{RESUMEN}

El artículo pretende contribuir a la definición del perfil docente en Educación Secundaria y acentuar su función pedagógica. Se trata de un estudio de revisión teórica y análisis descriptivo. Primero, se sintetiza el contexto normativo trazado para la educación hasta el 2030 por la UNESCO y la OECD. Después, se explora en profundidad la literatura científica buscando los núcleos fundamentales sobre los que construir un perfil común para el profesorado de secundaria. Tras un proceso de clasificación temática con sentido práctico, los contenidos se articulan en cuatro categorías fundamentales, fruto de la reflexión sobre las fuentes consultadas. El marco teórico resultante, formado por cuatro funciones y roles asociados, constituye un posible modelo de formación transdisciplinar docente. En la conclusión se propone la continuidad del debate sobre qué valor tiene el conocimiento pedagógico en el profesorado de secundaria; y cuáles son las implicaciones prácticas que se extraen del cuerpo teórico analizado.

Palabras claves: enseñanza secundaria, formación de profesores, formación inicial, profesión docente, rol del profesor.

\begin{abstract}
The article aims to contribute to the definition of the profile of teachers in Secondary Education and to emphasize its pedagogical function. It is a study of theoretical review and descriptive analysis. First, it's synthesized the normative context outlined for education until 2030 by UNESCO and the OECD. Then, the scientific literature is explored in depth, looking for the focal points on which to build a common profile for secondary school teachers. After a process of thematic classification with practical sense, the contents are divided into four fundamental categories, as consequences of reflection on the sources consulted. The resulting theoretical framework, consisting of four functions and associated roles, constitutes a possible model for transdisciplinary teacher training. In the conclusion, the continuity of the debate on the value of pedagogical knowledge in secondary school teachers is proposed; and what are the practical implications that are extracted from the theoretical body analyzed.
\end{abstract}

Key words: secondary education, teacher training, initial training, teaching profession, teacher role. 


\section{INTRODUCCIÓN}

La ampliación de contenidos curriculares e itinerarios de especialización, así como la innovación digital, están virtualizando los procesos educativos y transformando la formación del profesorado (Loughran y Hamilton, 2016). La visión disgregada del conocimiento y el utilitarismo nos conducen a una concepción parcial de la educación, de su organización y del rol del docente, que no está respondiendo al actual contexto internacional de demanda de sostenibilidad humanitaria y ambiental.

Los docentes tienen el inmenso poder y la responsabilidad de formar a los nuevos ciudadanos del mundo. Pueden avivar y estimular el talento o pueden apagarlo. El reto que ha inspirado este trabajo es transformar la cultura del aula, del centro educativo y, por extensión, del sistema, a partir de la formación de los docentes en líneas pedagógicas no deterministas, humanistas, inclusivas y respetuosas. Con esa finalidad, el primer objetivo es describir un proceso de reflexión sistemática y documentada sobre el perfil del profesorado y sus necesidades de formación. El segundo objetivo es aportar a la comunidad científica y profesional una herramienta que reactive el debate sobre la formación docente en la etapa de enseñanzas medias o educación secundaria.

La educación secundaria surge ligada a la formación superior durante la segunda mitad del siglo pasado. La finalidad preparatoria de esta etapa y su estructura en ciclos, modalidades o itinerarios optativos, condicionan las funciones de su profesorado (Lorenzo, Muñoz y Beas, 2015). Sin embargo, también se vincula a la enseñanza primaria en diversos aspectos, especialmente en los cursos obligatorios: en un currículo menos especializado que el de educación superior y en el sistema de contratación del profesorado (Vaillant, 2009). Por otro lado, son abundantes las referencias que critican el cambio de primaria a secundaria por el desfase cultural y metodológico entre etapas, pero, sobre todo, por las dificultades socioemocionales a las que se enfrenta su alumnado (Gairín, 2007; Galton, 2010; Hanewald, 2013; Monarca y Rincón, 2010) y por la escasa formación pedagógica de los docentes en justicia social, equidad y sostenibilidad (Sepúlveda, Brunaud y Carreño, 2016; Silva, Diniz y Zeichner, 2017).

Ser profesor de secundaria se ha convertido en una tarea muy difícil. Muchos profesionales se sienten incómodos y desmotivados, indefensos ante la complejidad del contexto y de la adolescencia. Su formación arrastra muchas controversias desde los comienzos y una amplia heterogeneidad internacional respecto a sentidos teóricos y organización práctica. La diferencia más sustancial se refiere al momento de la selección del profesorado, que puede ser antes, durante o después de adquirir la formación (Ries, Yanes y González, 2016).

Básicamente, se definen dos tipos de modelos formativos para el profesorado de secundaria en el ámbito internacional: los modelos concurrentes o simultáneos y los modelos consecutivos o sucesivos. También hay países donde coexisten los dos modelos. Cada tipología tiene su propio origen histórico, acentuándose la importancia de los contenidos en el concurrente y del proceso pedagógico en el consecutivo (Imbernon, 2019). Sin embargo, a pesar de ser necesaria la consideración de estas diferencias por su trascendencia para el desempeño práctico, el núcleo de interés principal para este trabajo es la visión transversal que se puede hacer de todos ellos. ¿Es posible trazar una hoja de ruta re-humanizadora de la formación docente, que sea pragmática y sostenible para todos los modelos de formación del profesorado? Desde un sentido transdisciplinar, es preciso dar 
respuesta a la demanda internacional de construir un futuro digno para todos, a partir de la educación en justicia social, inclusión y protección del medio ambiente.

La formación en la temprana juventud tiene poder auténtico para dotar al planeta, a corto plazo, de ciudadanos competentes, reflexivos y comprometidos. Y su profesorado es la piedra angular sobre la que se levanta ese futuro. Por tanto, las preguntas que guían este trabajo de investigación bibliográfica son: ¿Qué funciones han de constituirse como las prioritarias en el desempeño profesional de todo docente de secundaria? ¿Qué roles han de asumirse para el ejercicio de dichas funciones? ¿Cómo afecta ese escenario a la formación del profesorado?

\section{MÉTODO}

Se desarrolla un proceso de revisión documental y análisis de tipo descriptivo, para contextualizar y sintetizar conocimientos fragmentados. El propósito es actualizar el estado del tema, conocer las tendencias y líneas de investigación más extendidas y así poder aportar nuevas cuestiones de debate. La búsqueda y el análisis se llevan a cabo en dos fases:

a) Se analizan en profundidad todos los últimos documentos publicados por la OECD y la UNESCO, que contienen líneas de pensamiento y directrices con sentido para la Educación Secundaria hasta el 2030. Se trata de configurar un criterio normativo que sirva como referente para la siguiente fase, de selección de publicaciones científicas. La síntesis de esta Fase I de revisión documental y análisis descriptivo se desarrolla en el siguiente epígrafe.

b) Se realiza una búsqueda bibliográfica en las bases de datos ERIC, Web of Science y Google Scholar. Se introducen los siguientes descriptores (en inglés y español): secondary education, teaching methods, academic support, educational relationship, entrepreneurial education, experiential learning, learning outcomes, teaching models, teaching profession y teaching identity. Se trata de configurar un estado del arte actualizado en nuestro campo científico, que pueda responder a las actuales necesidades internacionales de transformación educativa y formación del docente de secundaria.

\section{RESULTADOS}

\subsection{MARCOS NORMATIVOS DE LA UNESCOY DE LA OECD PARA EL 2030}

Se analizan y sintetizan varias publicaciones de organismos internacionales de referencia en educación. Estas instituciones realizan periódicamente recomendaciones a los gobiernos nacionales y a sus respectivos departamentos con competencias en educación. Por ello, asumiendo que son referentes en la definición de la agenda ideológica y política, se consideran un marco práctico para orientar el posterior análisis de la literatura científica.

La OECD (2016), en su discurso para reforzar y revalorizar el capital humano en beneficio de la productividad global, acentúa una serie de competencias deseables para 
las nuevas generaciones de jóvenes. El interés prioritario recae sobre la competencia de autorregulación del aprendizaje a lo largo de la vida y el potencial de la comunicación. La mayoría de las habilidades mencionadas tienen un sentido preparatorio para el desempeño laboral en contextos dinámicos e internacionales: comunicación intercultural, pensamiento reflexivo y dominio de idiomas. Aunque se menciona con menor énfasis, el mismo informe también cita otras habilidades actitudinales y valores que debe promover la educación: flexibilidad o capacidad de adaptación, empatía, apertura y respeto intercultural, alteridad, mentalidad y responsabilidad global, así como revalorización de la dignidad humana.

Competencias como el pensamiento crítico, el trabajo colaborativo, la creatividad y la autorregulación, están adquiriendo tanto valor actualmente, que no se pueden obviar en los planes formativos de ninguna etapa (OECD, 2019a). Son las herramientas para convertir los aprendizajes específicos en funcionales y actuales. En definitiva, para producir nuevas ideas, teorías, productos y conocimientos.

En esta última referencia, en la que se detallan los resultados del Informe TALIS 2018 (OEDC, 2019a), el principal foco de interés es la figura del docente. A través de la amplitud y profundidad de los indicadores recopilados, TALIS tiene como objetivo examinar los factores que inciden en la profesionalidad del docente. Se desarrollan cinco pilares básicos que enriquecen la labor del profesorado y mejoran la calidad educativa: el conocimiento y las competencias necesarias para enseñar; las oportunidades profesionales; la cultura colaborativa entre docentes; el nivel de responsabilidad profesional y autonomía; y el prestigio percibido de la profesión.

En otro informe de la OECD (2019b) se destaca la importancia del conocimiento pedagógico de los docentes como ingrediente fundamental para una enseñanza eficaz y de alta calidad. Es preciso tener una formación profunda acerca de las ciencias de la educación, independientemente de lo que se enseñe. Los hallazgos de este trabajo de revisión sistemática van más lejos. Se afirma que el conocimiento profundo sobre pedagogía es un indicador del flujo compartido de experiencia docente entre profesionales de diferentes disciplinas y diversos entornos de enseñanza. Se subraya la importancia que tiene el disfrute en la labor del profesorado y el potencial de ejercer un liderazgo profesional personalizado y autodeterminado.

Como la organización líder que encabeza la definición de los Objetivos de Desarrollo Sostenible (ODS) y la Agenda de Educación 2030, la UNESCO ha publicado varios documentos en los últimos años con líneas de acción muy claras. En "The Incheon Declaration and Framework for Action" (UNESCO, 2015a) y "Rethinking Education: Towards a global common good" (UNESCO, 2015b), la UNESCO sitúa el desarrollo educativo como el principal recurso hacia una mayor justicia social e igualdad al servicio de una humanidad común. La clave se encuentra en lograr un mayor sentido de empoderamiento para el alumno, especialmente cuando está alineado con el desarrollo de la capacidad humana, en beneficio del emprendimiento y la autorregulación competencial.

La UNESCO (2016) se propone transformar las cualificaciones profesionales hacia visiones interdisciplinares y transdisciplinares, que no coinciden con las tradicionales materias curriculares. Se critica la visión limitada de las áreas académicas tradicionales por estar al servicio de necesidades pertenecientes a otro tiempo pasado. Respecto a la formación humanizada e individualizada, se recomienda la orientación para el desarrollo de la carrera para lograr perfiles personalizados, versátiles y en constante evolución. Se insiste en que los contextos educativos no toleren ningún tipo de violencia, abuso o rechazo 
a otros seres humanos. Por último, se destaca la competencia digital con sentido analíticocrítico, para gestionar la información multicanal y su accesibilidad.

En la difusión que realiza la UNESCO (2017) en cuanto al liderazgo y la promoción de la agenda de Educación 2030, se vinculan los retos educativos a los 17 Objetivos de Desarrollo Sostenible (ODS). Se prioriza la concentración de esfuerzos para lograr el ODS 4: "Garantizar una educación inclusiva y equitativa de calidad y promover oportunidades de aprendizaje durante toda la vida para todos". Una vez más, su mayor énfasis se dirige hacia la transformación de la educación para lograr el desarrollo sostenible. El único modo es preparar a los educandos para tomar decisiones informadas y actuar con responsabilidad a favor de la integridad ambiental, la viabilidad socio-económica y la justicia social.

La urgencia de los desafíos que tenemos ante nosotros requiere una transformación radical de nuestras concepciones sobre el conocimiento, el aprendizaje y la educación. El auge de las nuevas tecnologías, incluida la inteligencia artificial y el big data, también plantea importantes preocupaciones éticas y políticas, especialmente porque la innovación y cambio tecnológico tienen un historial de incremento de las desigualdades humanas. El proyecto Futures of Education de la UNESCO (2019) es una oportunidad para movilizar la inmensa diversidad que tenemos a nivel mundial respecto al ser y al saber sobre educación. De nuevo se abre la puerta a la reflexión y al debate sobre cómo podría ser necesario repensar la educación en un mundo de creciente complejidad, incertidumbre y precariedad.

Tanto la UNESCO como la OECD establecen marcos normativos claros para transformar la educación en todo el mundo, con el propósito de extraer lo mejor de la globalización sin olvidarnos de preservar la sostenibilidad. Sin embargo, si bien la articulación de estas normas puede parecer convergente de alguna manera, hay evidencias de divergencia política entre las dos organizaciones que no pueden obviarse si se profundiza en el análisis. En línea con los objetivos de este trabajo, se dirige el foco de atención únicamente hacia las áreas de convergencia en relación a todos los aspectos valorados. En esencia, se cuestiona: ¿Puede la educación preparar a los estudiantes del siglo XXI para tener éxito individual y contribuir al progreso de la economía global, al mismo tiempo que cultiva y revaloriza una humanidad compartida que sea digna para todos?

\subsection{PERFIL TRANSDISCIPLINAR DEL PROFESORADO DE SECUNDARIA}

A partir de la selección y análisis riguroso de las fuentes científicas consultadas, se constituye un cuerpo de reflexión teórica respecto al perfil común/transversal de todo el profesorado de secundaria como respuesta al marco normativo. Se describen y fundamentan cuatro funciones con sentido pedagógico y valor para la profesión. Cada función nos remite a un rol docente asequible desde la formación inicial y continua (Figura 1). Son cuatro visiones parciales y complementarias de un perfil complejo por naturaleza, que dirigen la mirada hacia lo esencial del proceso educativo según las recomendaciones para esta década. 
Estudios Pedagógicos XLVII N² 2: 215-229, 2021

CÓMO FORMAR AL PROFESORADO DE SECUNDARIA PARA SATISFACER LAS NECESIDADES EDUCATIVAS DE LA PRÓXIMA DÉCADA

Figura 1. Construcción del perfil transdisciplinar del profesorado: Funciones y Roles.

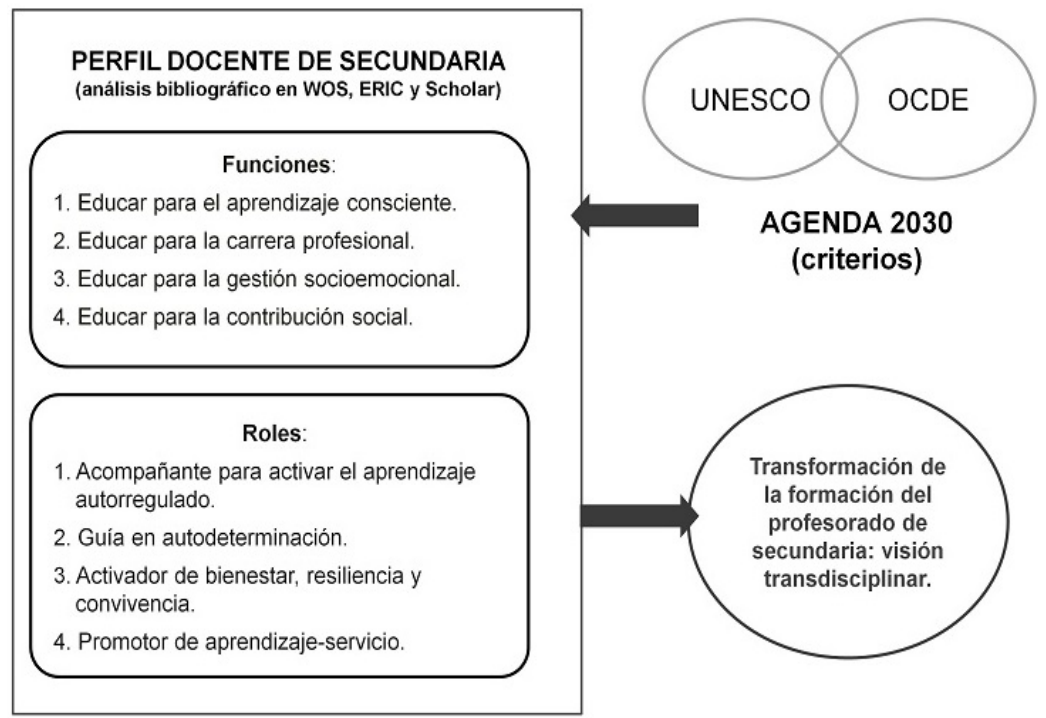

Realización propia.

3.2.1. Educar para el aprendizaje consciente: el rol de activador del aprendizaje autorregulado

Esta función se refiere a la toma de conciencia sobre el aprendizaje y a la capacidad para contextualizarlo en relación a su sentido funcional. Se trata de prevenir las dificultades encontradas en estudiantes universitarios cuando se les demandan actividades metacognitivas de reflexión oral y escrita sobre su aprendizaje (Landis, Scott y Kahn, 2015; Morreale, Van Zile-Tamsen, Emerson y Herzog, 2017). El profesorado debe facilitar las herramientas para educarse con autonomía y autoevaluarse. La finalidad última es ser competentes para deconstruir, co-construir y reconstruir el conocimiento (Alsina, Batllori, Falgàs y Vidal, 2019). Es una función muy significativa que se asocia con la demanda de profesionales con alta cualificación y perfiles orientados a la innovación tecnológica y la investigación.

El profesor de secundaria debe saber cómo crear contextos de formación que fomenten la construcción de proyectos motivadores y abiertos. El objetivo educativo es el aprendizaje auto-regulado, que se relaciona con el aprendizaje efectivo a lo largo de la vida y se compone de tres factores: metacognición, motivación y conocimiento estratégico (De Smul, Heirweg, Van Keer, Devos y Vandevelde, 2018). Con el fin de convertirse en estudiantes autorregulados, los alumnos necesitan dominar una serie de estrategias de aprendizaje generalizables a múltiples contextos y necesidades, a partir de aprendizajes específicos en situaciones concretas (Kistner, Rakoczy, Otto, Klieme y Büttner, 2015). Pero, además, el profesorado debe focalizar su metodología hacia el desarrollo de las competencias que posibilitan la cultura emprendedora: tener iniciativa, buscar oportunidades, valorar ideas, movilizar recursos, planificar y gestionar, enfrentar incertidumbres y riesgos, etc. 
Los métodos que facilitan desarrollar estas habilidades tienen el denominador común de pertenecer al grupo de las metodologías activas (Paños, 2017). Sin embargo, utilizar cualquiera de ellas no es suficiente: el buen profesor tiene como punto de partida la inteligencia cultural, en la que se integran lo académico, lo práctico y lo comunicativo, para lograr un aprendizaje dialógico y comprometido (Prieto y Duque, 2009).

El rol docente más eficaz se define como activador de la implicación personal en progresivos retos problemáticos, empresariales, investigadores, etc. (Hattie, 2008). Es pertinente, entonces, difundir la idea de "educar para educarse y no educar para ...", estimulando la competencia y predisposición de los alumnos/as a continuar aprendiendo durante toda la vida (Belanger y Federighi, 2000). Esta propuesta se fundamenta también en el concepto de empoderamiento individual y de grupo, lo que conduce a transformar la concepción actual del docente "transmisor de conocimientos" en un modelo de profesorado que realiza acompañamiento personalizado y desarrolla una cultura de autoevaluación del aprendizaje (Del Corso y Rehfuss, 2011; Lengelle, Meijers, Poell y Post, 2014).

Ahora bien, para ejercer este rol de acompañante implicado, es imprescindible el contacto humano directo y mantenido en el tiempo. Es preciso, por tanto, reflexionar sobre la progresiva sustitución que se está haciendo de la intervención personal por la tecnología. También, acerca de que cada comienzo de curso se emprende una nueva etapa de conocimiento, de relación y de trabajo con alumnos nuevos; y este proceso se multiplica por varios grupos en los que cada profesor imparte su docencia (Santos Guerra, 2009).

\subsubsection{Educar para la carrera profesional: el rol de guía en autodeterminación}

El aprendizaje de autodeterminación se fundamenta en el proceso de construcción flexible y evolutiva de un proyecto personal que integre todas las áreas que conforman la identidad. Se comienza con la toma de conciencia respecto a la variabilidad de determinantes que condicionan nuestras vidas, como fuente de narrativa individual en evolución emergente. Y se continúa con la consideración de que el proceso es social y tiene que ser ecológico para lograr su sostenibilidad (Do Céu Teveira y Rodríguez Moreno, 2010; Plant, 2015). Se trata de proyectarse uno mismo en un futuro inmediato y un poco más lejano, expresando reflexivamente cómo se desea configurar la trayectoria formativa y profesional con el paso de los años (Irving y Malik, 2005; Romero, 2009).

La idea de carrera versátil, dinámica y sin fronteras, como proceso de creación de una identidad personal y social compleja desde la escuela, se ha reforzado en la literatura especializada durante los últimos años de forma exponencial (Lo Presti, 2009; Romero, Seco y Lugo, 2015), desarrollándose un campo que conecta la psicología del trabajo, la orientación vocacional y la educación por competencias. Lo esencial es que la intervención facilite al individuo acercarse a su propio proyecto de vida teniendo en cuenta todo su sistema (familiar, contextual, ...) extendiendo las acciones pedagógicas sobre todas sus partes (Kettunen \& Tynjälä, 2018).

El docente tiene que estimular la reflexión: guiar las narrativas sobre los proyectos, su expresión y el aprovechamiento del apoyo de orientadores, profesores, compañeros y familiares. Algunos modelos de intervención afines, que pueden tomarse como ejemplos de actuación, son: el asesoramiento para la construcción del proyecto profesional, el coaching, el desarrollo de competencias transversales, la formación específica para aumentar la empleabilidad y la concienciación hacia la igualdad de oportunidades. Se han 
de tener presentes las estrategias transformadoras de los educandos y de los educadores, puesto que desde ese equilibrio y mediante el trabajo cooperativo, se puede conseguir un mejor aprendizaje para la autodeterminación vital (Cortés, Rodríguez y Val, 2018). En este sentido, las acciones de acompañamiento con carácter vocacional pueden integrarse con actividades que también favorezcan la equidad, inclusión y convivencia (Sandoval, Simón y Echeita, 2019).

Del análisis realizado emerge un propósito básico: lograr que el desarrollo profesional se comprenda como un proceso extensible a toda la vida y que comienza en edades tempranas. La toma de decisiones y su evaluación consciente son susceptibles de aprendizaje a través de experiencias educativas sencillas y cotidianas (Acevez, Santos y Valenzuela, 2014; Olmos, 2014). Desde esta perspectiva, es esencial que el alumnado establezca sus propias estrategias para tomar decisiones desde un estilo reflexivo-no impulsivo, con conciencia de sí mismos y de su entorno, en lo relativo a competencias, valores, rendimiento, limitaciones, etc. La intervención del profesorado no puede ser determinante, sino orientada a estimular la apertura y creatividad individual (Draaisma, Meijers y Kuijpers, 2018).

\subsubsection{Educar para la gestión socioemocional: el rol de activador de bienestar, resiliencia y convivencia}

Un concepto ampliamente vinculado en las referencias documentales con la regulación del aprendizaje y la toma de decisiones es la gestión socioemocional (Izquierdo, 2013; Di Giusto, Martín, Arnaiz y Guerra, 2014; Zhoc, Chung y King, 2018). Se enfatiza la importancia de la afectividad, de los valores éticos y de las influencias sociales en la toma de decisiones. La educación en inteligencia emocional se considera una formación competencial básica relacionada directamente con el logro académico y el bienestar en contextos académicos (Goetz y Bieg, 2016).

Por otro lado, la autorregulación social y afectiva es un factor protector de conductas violentas, impulsividad y desajuste emocional (Pena y Repetto, 2008; Garaigordobil y Oñederra, 2010). Minimiza el impacto emocional de los sucesos negativos y repara la estabilidad personal en los adolescentes. Los sujetos emocionalmente más inestables carecen de recursos para frenar su impulsividad y son más propensos a no usar conductas prosociales (Mestre, Samper y Frías, 2002; Tur, Mestre, Samper y Malonda, 2012). Los datos sobre violencia en los centros educativos informan de que un $9.3 \%$ ha sufrido acoso y un $6.9 \%$ ciberacoso, según el informe "Yo a eso no juego" de Save the Children (Nocito, 2017).

Es necesaria la formación del profesorado en relación a la gestión socio-afectiva del grupo y el fomento de relaciones interpersonales adecuadas (Postigo, González, Mateu y Montoya, 2012; Sánchez y Cerezo, 2011). Por ello, el profesorado se convierte en el punto estratégico a considerar en las dinámicas escolares; como modelo y facilitador de relaciones interpersonales estables y positivas (Casas et al., 2008).

El profesorado de secundaria ha de poseer un bagaje propio sólido en competencias emocionales (Mondal y Saha, 2017). Las razones son recíprocas: como aspecto esencial del desarrollo profesional del docente y, por extensión, para potenciar su desarrollo en el alumnado. El reto es que todo el profesorado sea competente en pensamiento emocional y tenga una sólida autoestima. Son la base para contagiar actitudes positivas y para ser buen profesor en su disciplina específica (Güell y Muñoz, 2003; Segura y Arcas, 2003). En un estudio reciente realizado con una amplia muestra alemana, la extraversión y el 
entusiasmo por la enseñanza son predictores del aprendizaje de los alumnos. Y el disfrute con la enseñanza, así como su toma de conciencia, son predictores significativos de mejora de disciplina y convivencia en el aula (Baier et al., 2018).

Por otro lado, para educar en resiliencia y bienestar parece pertinente recuperar el concepto de fluir como camino para lograr la felicidad y encontrar la motivación en la rutina diaria de las aulas (Csikszentmihaly, 1997; Dos Santos et al., 2018). Las condiciones del flujo psicológico ofrecen un marco de sugerencias para la acción que se pueden aplicar al trabajo intelectual, la actividad física, las relaciones sociales, etc. El rol del profesorado es el de dinamizador de experiencias de aprendizaje que permitan a los estudiantes aprender $a$ fluir. Y su enfoque personal de la profesión se sitúa en esa misma línea humanista y positiva (Harris, 2013; López, 2013).

\subsubsection{Educar para la contribución social: el rol de promotor de aprendizaje-servicio}

La cuarta y última función se refiere a la transferencia del aprendizaje, en respuesta a los retos propuestos por las instituciones europeas en relación a la responsabilidad social y medioambiental de la educación. La educación, a través del cuidado de las relaciones, del clima y del estilo educativo, transforma tanto la experiencia de aprendizaje como la actitud y estilo educativo del docente (García y Mendía, 2015). Es necesario avanzar en programas que se apoyen en todas las redes de aprendizaje existentes en la comunidad y quebrar las formas tradicionales de entender la práctica profesional docente hacia la reconstrucción de su saber práctico (Mayor y Rodríguez, 2016).

En varios estudios, tanto de meta-análisis como de desarrollo de casos en profundidad, se sugiere que una pedagogía crítica de aprendizaje-servicio alienta a los estudiantes a pensar más profundamente y desarrollar compromisos para actuar por la justicia social. También se constata la percepción de mayor autoeficacia y satisfacción, tanto en el alumnado como en los docentes (Mitchell, 2007; Uribe, 2018; Wasner, 2019).

Además, la participación en programas de aprendizaje-servicio de alta calidad se relaciona con mayor asistencia a la escuela secundaria, calificaciones más altas, mayores habilidades para resolver problemas y una mejor adquisición de conocimientos relacionados con lengua, matemáticas, ciencias y estudios sociales (Billig, 2004; Puig, Gijón, Martín y Rubio, 2011). En definitiva, uno de los objetivos fundamentales de esta metodología es la adquisición de contenidos curriculares, sólo que, de forma integrada, contextualizada en la realidad y orientada a la acción.

El docente adquiere un rol de diseñador y promotor de comunidades de aprendizaje en torno al programa de aprendizaje-servicio, adoptando relaciones democráticas horizontales con el alumnado (Ochoa, Pérez y Salinas, 2018). Su principal función es la de dinamizador de participación auténtica y negociada, activando el aprendizaje del alumnado, en lugar de sólo facilitarlo. Este rol docente se amplía con sentido práctico en el Cuadro 1 (Mayor, 2018; Mendía, 2013). 
Cuadro 1. Estrategias para la práctica del rol docente en APS. Adaptación

\begin{tabular}{|l|l|l|}
\hline Conocimiento & Actuación & Actitud \\
\hline $\begin{array}{l}\text { Familiarizarse con } \\
\text { los conocimientos, } \\
\text { habilidades, fortalezas } \\
\text { e intereses de cada } \\
\text { estudiante. }\end{array}$ & $\begin{array}{l}\text { Cuestionar a los alumnos } \\
\text { durante todo el proceso } \\
\text { para ayudarles a reflexionar } \\
\text { sobre cómo y por qué se } \\
\text { generan los cambios. }\end{array}$ & $\begin{array}{l}\text { Ofrecer opciones } \\
\text { de planificación, } \\
\text { implementación y } \\
\text { evaluación al alumnado. }\end{array}$ \\
\hline $\begin{array}{l}\text { Investigar la conexión } \\
\text { entre conocimientos } \\
\text { disciplinarios y } \\
\text { programas de APS. }\end{array}$ & $\begin{array}{l}\text { Problematizar para } \\
\text { estimular el pensamiento } \\
\text { crítico y la creatividad. }\end{array}$ & $\begin{array}{l}\text { Estimular competencia } \\
\text { y confianza a la vez, con } \\
\text { un incremento progresivo } \\
\text { del desafío. }\end{array}$ \\
\hline $\begin{array}{l}\text { Explorar los recursos } \\
\text { de la comunidad y las } \\
\text { necesidades del entorno. }\end{array}$ & $\begin{array}{l}\text { Realizar una evaluación } \\
\text { no intrusiva, cuya } \\
\text { finalidad última sea que } \\
\text { los estudiantes lleguen } \\
\text { a ser competentes en } \\
\text { autoevaluación. }\end{array}$ & $\begin{array}{l}\text { Promover sentimientos } \\
\text { de pertenencia a la } \\
\text { comunidad; una cultura } \\
\text { de respeto, cuidado, } \\
\text { imparcialidad y } \\
\text { responsabilidad, actuando } \\
\text { como modelo. }\end{array}$ \\
\hline
\end{tabular}

Las nuevas generaciones deben aprender a cuidar a las personas y al medio natural; y desarrollar voluntad para hacerlo. Al ejercitarnos en el amor por los otros, incluso por los que no consideramos parecidos a nosotros mismos, ni dignos de dicho amor, se transforma la fisiología del cerebro, aumentando el área de la felicidad y desactivándose la del miedo. La compasión es una competencia, es educable y tiene potencial para reestructurar el cerebro humano (Davidson y McEwen, 2012). En sintonía con esta idea, Damasio (2018) aconseja trabajar por la felicidad de otras personas para lograr nuestra propia felicidad. Ambos propósitos deben estar articulados y resultar una motivación única si realmente queremos ser felices.

\section{CONCLUSIONES}

Para finalizar el artículo, se aborda la cuestión urgente de dirigir la formación del profesorado de enseñanzas medias hacia una reconceptualización transdisciplinar y rehumanizadora de su perfil. Se reflexiona sobre las posibilidades de potenciar lo que todos los docentes, especialistas cada uno en su materia, tienen en común: su sentido educativo. Se trata de acercarles más a la figura del orientador, sin que por ello se distancien de su didáctica específica. Las conclusiones pretenden resultar de interés para los profesores universitarios que imparten la Formación del Profesorado de Educación Secundaria en todas sus ramas, así como para la formación permanente de los docentes en activo.

La primera reflexión que surge del análisis nos conduce a un dilema sobre la naturaleza de la innovación que se necesita para transformar la formación del profesorado. Las recomendaciones de los organismos internacionales analizados, especialmente las de la 
UNESCO, se refieren a una visión de la educación desde un enfoque cualitativamente distinto del que fundamenta el actual flujo de innovación en los planes formativos del profesorado. Desde un punto de vista hermenéutico, es cuestión de ascender en el enfoque desde el que se contempla el propio fin de la educación secundaria. El gran reto es transformar los planes educativos a partir de lo que realmente tiene valor para mejorar la vida de las personas y de la sociedad en su conjunto, hallando sinergias entre el desarrollo intelectual y la rehumanización de la educación al servicio personal y social.

Son muy abundantes las publicaciones y acciones innovadoras sobre estrategias didácticas y recursos. Por lo que la formación del profesorado suele enfocarse hacia la adquisición de habilidades para utilizar dichos recursos. Se incrementa cada vez más la diversidad de opciones metodológicas, como si de un menú a la carta se tratase. Pero ¿es adecuada la idea de eficacia de la enseñanza que se transmite con ese enfoque de la innovación? ¿Son conscientes los profesores de qué sentido pedagógico tienen las herramientas metodológicas, materiales, medios tecnológicos, etc. que están aplicando en sus aulas? Y lo que es más preocupante, ¿se contemplan las cualidades humanas, actitudinales y comunicativas que han de poseer los docentes para poder ejercer desde las recomendaciones propuestas?

El desarrollo de las cuatro funciones descritas en este artículo redirige la mirada del formador del profesorado hacia cuatro sentidos viables y sostenibles, con el apoyo de las referencias aportadas. La investigación educativa está actualmente avanzando en la definición del perfil docente, de sus elementos y de los procesos formativos que facilitan su desarrollo (Alsina et al., 2019; Cantón y Tardiff, 2018). Además, se ha revalorizado recientemente la toma de conciencia reflexiva sobre el rol profesional durante el periodo de formación inicial, tanto en la formación de Grado como de Máster, con el sentido de motivar y aumentar el compromiso de los futuros educadores (Gravett et al., 2017; Loughran y Hamilton, 2016). "¿Qué ocurre con el profesorado que ha olvidado su historia?" se preguntan Borbar y López (2020, p. 231), al relacionar la necesidad de conocer la realidad interior, vinculada a la subjetividad, con la sensibilidad educativa.

Por ello, si la aspiración es formar a un profesorado que acompañe a su alumnado hacia el aprendizaje autónomo, funcional y con sentido social, que humanice la acción educativa, la diversifique hacia nuevos retos y promueva el bienestar psicosocial (López, 2013; Mendía, 2013), no se puede diseñar su formación desde un modelo de adquisición de conocimientos teóricos y de estrategias a la carta. La formación del profesorado ha de generar contextos reflexivos, estimulantes, afectivos y dilemáticos.

Sin embargo, un gran obstáculo difícil de salvar es la visión disciplinar todavía vigente en la formación universitaria. En las facultades donde se gradúan los que serán profesores de ciencias, matemáticas, lengua, idiomas, etc., el conocimiento se fracciona, es enciclopédico, descontextualizado respecto a sus condiciones de construcción y absolutista. La visión disgregada del conocimiento y el utilitarismo nos conducen a una concepción parcial de la educación, de su organización y del rol del docente. (Harris, 2013). La formación del profesor de secundaria puede mejorar adaptando los contenidos y metodología a los actuales paradigmas socio-educativos y revalorizando su rol como educador para la vida (Perrenoud, 2012). Sin embargo, el núcleo de la transformación, segunda conclusión de este análisis se encuentra en la modificación cualitativa de la visión que se tiene de la profesión: de una suma de conocimientos específicos y didácticos a un modelo de profesor y un saber profesional con entidad propia y genuina (Carnicer y Furió, 2002; Imbernón, 2019). 
El principal reto está en reclutar para la docencia a titulados valiosos. Es prioritario mejorar su condición laboral, social y económica, para que la profesión docente resulte una carrera atractiva y con prospectiva de crecimiento personal. La formación pedagógica tiene de resultar estimulante y generar proyectos atractivos; porque las personas con talento necesitan contextos dinámicos en los que desarrollar su potencial (Hattie, 2008) y retos profesionales exigentes. La tercera conclusión nos lleva a proponer una premisa básica, si lo que se quiere es contar con los mejores profesionales al servicio de la educación: aumentar la exigencia tanto en la selección como en la evaluación del profesorado.

Este trabajo aporta una estructura teórica para continuar reflexionando sobre esas representaciones, con el propósito de introducir una nueva mirada hacia "lo genuino" de la educación secundaria y de la formación de su profesorado, aprovechando la agenda marcada por los objetivos internacionales marcados para el 2030. Es preciso continuar mediante una investigación de meta-análisis más exhaustiva y rigurosa desde el punto de vista empírico.

\section{REFERENCIAS BIBLIOGRÁFICAS}

Acevez, N., Santos, D. y Valenzuela, J. R. (2014). Competencia transversal para desarrollar planes de vida personales: caracterización de estudiantes expertos. Revista de Investigación Educativa de Graduados en Educación, 5(9), 21-29.

Alsina, Á., Batllori, R., Falgàs, M. y Vidal, I. (2019). Marcas de autorregulación para la construcción del perfil docente durante la formación inicial de maestros. Revista Complutense de Educación, 30(1), 55-74.

Baier, F., Decker, A. T., Voss, T., Kleickmann, T., Klusmann, U. \& Kunter, M. (2018). What makes a good teacher? The relative importance of mathematics teachers' cognitive ability, personality, knowledge, beliefs, and motivation for instructional quality. British Journal of Educational Psychology 89(4), 767-786.

Belanguer, P. \& Federighi, P. (2000). Unlocking people's creative forces: a transnational study of adult learning policies. Hamburgo: UNESCO Publishing.

Billig, S. H. (2004). Heads, hearts, and hands: The research on K-12 service-learning. Growing to Greatness. St. Paul: National Youth Leadership Council.

Borbar, V. y López, A. (2020). Lo personal en lo educativo. Revista Interuniversitaria de Formación del profesorado, 95(34.1), 227-242.

Cantón, I. y Tardiff, M. (Coords.) (2018). Identidad profesional docente. Madrid: Narcea.

Carnicer, J. y Furió, C. (2002). La epistemología docente convencional como impedimento para el cambio. Revista Investigación en la Escuela, 47, 33-52.

Casas, F., González, M., Carme, M., Navarro, D., Malo, S., Figuer, C. y Bertrán, I. (2008). Informe sobre experiencias de participación social efectiva de niños, niñas y adolescentes. Madrid: Ministerio de Educación, Política Social y Deporte.

Cortés, A., Rodríguez, A. y Val, S. (Coords.) (2018). Estrategias transformadoras para la educación. Madrid: Pirámide.

Csikszentmihalyi, M. (1997). Fluir. Una psicología de la felicidad. Barcelona: Kairós.

Damasio, A. (2018). El error de Descartes: la emoción, la razón y el cerebro humano. Barcelona, Ediciones Destino.

Davidson, R. J. \& McEwen, B. S. (2012). Social influences on neuro-plasticity: Stress and interventions to promote well-being. Nature Neuroscience, 15, 689-695.

Del Corso, J. \& Rehfuss, M. C. (2011). The role of narrative in career construction theory. Journal of Vocational Behavior, 79(2), 334-339. 
De Smul, M., Heirweg, S., Van Keer, H., Devos, G. \& Vandevelde, S. (2018). How competent do teachers feel instructing self-regulated learning strategies? Development and validation of the teacher self-efficacy scale to implement self-regulated learning. Teaching and teacher education, $71,214-225$.

Di Giusto, C., Martín, M. E., Arnaiz. A. y Guerra, P. (2014). Competencias personales y sociales en adolescentes. Revista Iberoamericana de Educación, 66, 89-104.

Diniz-Pereira, J. E. \& Zeichner, K. M. (2017). A pesquisa na formação e no trabalho docente. Autêntica.

Do Céu Teveira, M. y Rodríguez Moreno, M. L. (2010). La gestión personal de la carrera y el papel de la orientación profesional. Teoría, práctica y aportaciones empíricas. REOP, 21(2), 335-345.

Dos Santos W. O., Bittencourt, I. I., Dermeval, D., Isotani, S., Marques, L. B. \& Silveira, I. F. (2018). Flow Theory to Promote Learning in Educational Systems: Is it Really Relevant? Brazilian Journal of Computers in Education - RBIE, 26(2), 29-59.

Draaisma, A., Meijers, F. \& Kuijpers, M. (2018) The development of strong career learning environments: the project 'Career Orientation and Guidance' in Dutch vocational education. Journal of Vocational Education \& Training, 70(1), 27-46.

Gairín, J. (2007). El reto de la transición entre etapas educativas. En S. Antúnez et al. La transición entre etapas. Reflexiones y prácticas (pp. 31-40). Barcelona: Graó.

Galton, M. (2010). Moving to Secondary School: What do Pupils in England Say About the Experience. En D. Jindal Scape (Ed.). Educational Transitions. Moving Stories from Around the Word (pp. 107-124). New York: Routledge.

Garaigordobil, M. y Oñederra, J. A. (2010). Inteligencia emocional en las víctimas de acoso escolar y en los agresores. European Journal of Educational and Psychology, 3(2), 243-256.

García, A. y Mendía, R. (2015). Acompañamiento educativo: el rol del educador en aprendizaje y servicio solidario. Profesorado. Revista de Currículum y Formación de Profesorado, 19(1), 42-58.

Goetz T. \& Bieg M. (2016) Academic Emotions and Their Regulation via Emotional Intelligence. In: Lipnevich A., Preckel F. \& Roberts R. (Eds.). Psychosocial Skills and School Systems in the 21st Century: Theory, research and practice (pp. 279-298). Springer International Publishing.

Gravett, S., De Beer, J., Odendaal Kroon, R. \& Merseth, K. K. (2017). The affordances of case-based teaching for the professional learning of student-teachers. Journal of Curriculum Studies, 49(3), 363-390.

Güell, M. y Muñoz, J. (Coords.) (2003). Educación emocional. Programa para la educación secundaria postobligatoria. Barcelona: Praxis.

Hanewald, R. (2013). Transition Between Primary and Secondary School: Why it is Important and How it can be Supported. Australian Journal of Teacher Education, 38(1), 62-74.

Harris, D. E. (2013). Applying Theory to Practice: Putting Deleuze to Work. International Journal of Sociology of Education, 2(2),142-166.

Hattie, J. (2008). Visible learning: A synthesis of over 800 meta-analyses relating to achievement. Abingdon: Routledge.

Imbernón, F. (2019). La formación del profesorado de educación secundaria: la eterna pesadilla. Revista de curriculum y formación del profesorado, 23(3), 151-163.

Irving, B. y Malik, B. (Eds.) (2005). Reflexiones Críticas sobre Educación para la Carrera y Orientación. Impulsando la justicia social en una sociedad globalizada. Londres: RoutledgeFalmer.

Izquierdo, M. (2013). La química ¿emociona? En V. Mellado, L. J. Blanco, A.B. Borrachero y J. A. Cárdenas (Eds.). Las Emociones en la Enseñanza y el Aprendizaje de las Ciencias y las Matemáticas (pp. 307-328). Badajoz: DEPROFE.

Kettunen, J. \& Tynjälä, P. (2018). Applying phenomenography in guidance and counselling research, British Journal of Guidance \& Counselling, 46(1), 1-11. 
Estudios Pedagógicos XLVII N ${ }^{\circ}$ 2: 215-229, 2021

CÓMO FORMAR AL PROFESORADO DE SECUNDARIA PARA SATISFACER LAS NECESIDADES EDUCATIVAS DE LA PRÓXIMA DÉCADA

Kistner, S., Rakoczy, K., Otto, B., Klieme, E. \& Büttner, G. (2015). Teaching learning strategies: The role of instructional context and teacher beliefs. Journal for Educational Research Online, 7(1), 176-197.

Landis, C., Scott, S. \& Kahn, S. (2015). Examining the role of reflection in ePortfolios: A case study. International Journal of ePortfolio, 5(2), 107-121.

Lengelle, R., Meijers, F., Poell, R. \& Post, M. (2014). Career writing: Creative, expressive and reflective approaches to narrative identity formation in students in higher education. Journal of Vocational Behavior, 85(1), 75-84.

López, E. (2013). Aproximación a la percepción y satisfacción del profesor tutor de Secundaria Obligatoria respecto a su labor. Revista de Investigación en Educación, 11(1), 77-96.

Lo Presti, A. (2009). Snakes and ladders: stressing the role of meta-competencies for post-modern careers. International Journal for Educational and Vocational Guidance, 9(2), 125-134.

Lorenzo, J. A., Muñoz, I. M. y Beas, M. (2015). Modelos de formación inicial del profesorado de Educación Secundaria en España desde una perspectiva Europea. Revista Complutense de Educación, 26(3), 741-757.

Loughran, J. \& Hamilton, M. L. (2016). International handbook of teacher education (Vol. 1). Gateway East, Singapore: Springer.

Mayor, D. (2018). Prácticas innovadoras de Aprendizaje-Servicio que promueven el enganche de los menores en la mejora de su comunidad. INNOVA Research Journal, 3(9), 63-75.

Mayor, D. y Rodríguez, D. (2016). Aprendizaje-servicio y práctica docente: una relación para el cambio educativo. Revista de Investigación Educativa, 34(2), 535-552.

Mendía, R. (2013). Guía de aprendizaje y servicio solidario: el acompañamiento educativo. Bilbao: Zerbikas Fundazioa.

Mestre, M. V., Samper, P. y Frías, M. D. (2002). Procesos cognitivos y emocionales predictores de la conducta prosocial y agresiva: La empatía como factor modulador. Psicothema, 14(2), 227- 232.

Mitchell, T. D. (2007). Critical service learning as social justice education: A case study of the citizen scholars program. Equity \& Excellence in Education, 40(2), 101-112.

Monarca, H. y Rincón, J. (2010). Tránsito a la ESO, ¿continuidad o ruptura? Cuadernos de Pedagogía, $401,28-31$.

Mondal, A. \& Saha, B. (2017). Job Satisfaction of Secondary School Teachers in Relation to Personality and Emotional Intelligence. American Journal of Educational Research, 5(10), 1097-1101.

Morreale, C., Van Zile Tamsen, C., Emerson, C. A. \& Herzog, M. (2017). Thinking skills by design: Using a capstone ePortfolio to promote reflection, critical thinking, and curriculum integration. International Journal of ePortfolio, 7(1), 13-28.

Nocito, G. (2017). Investigaciones sobre el acoso escolar en España: implicaciones psicoeducativas. REOP, 28(1), 104-118.

Ochoa, A. Pérez, L. M. y Salinas, J. J. (2018). El aprendizaje-servicio (APS) como práctica expansiva y transformadora. Revista Iberoamericana de Educación, 76, 15-34.

OECD (2016). Global Competency for an Inclusive World. Paris: OEDC Publishing.

. (2019a). New insights on teaching and learning: Contributions from TALIS 2018, Teaching in Focus. Paris. OECD Publishing.

. (2019b). The relevance of general pedagogical knowledge for successful teaching: Systematic review and meta-analysis of the international evidence from primary to tertiary education. Paris: OEDC Publishing.

Olmos, P. (2014). Competencias básicas y procesos perceptivos: factores claves en la formación y orientación de los jóvenes en riesgo de exclusión educativa y sociolaboral. Revista de Investigación Educativa, 32(2), 531-546.

Paños, J. (2017). Educación emprendedora y metodologías activas para su fomento. Revista Electrónica Interuniversitaria de Formación del Profesorado, 20(3), 33-48. 
Pena, M. y Repetto, E. (2008). Estado de la investigación en España sobre inteligencia emocional en el ámbito educativo. Revista Electrónica de Investigación Psicoeducativa, 6(2), 400-420.

Perrenoud, P. (2012). Cuando la escuela pretende preparar para la vida: ¿Desarrollar competencias o enseñar otros saberes? Barcelona: Graó.

Plant, P. (2015). Green guidance: guidance for the future. REOP, 26(1), 115 - 123.

Postigo, S., González, R., Mateu, C. \& Montoya, I. (2012). Predicting bullying: maladjustment, social skills and popularity. Educational Psychology, 32(5), 627-639.

Prieto, O. y Duque, E. (2009). El aprendizaje dialógico y sus aportaciones a la teoría de la educación. Revista Electrónica Teoría de la Educación, 10(3), 7-30.

Puig, J. M., Gijón, M., Martín, X. y Rubio, L. (2011). Aprendizaje-servicio y Educación Para la ciudadanía. Revista de Educación, $n^{\circ}$ extraordinario 2011, 45-67.

Ries, F., Yanes, C. \& González, R. (2016). A Study of Teacher Training in the United States and Europe. The European Journal of Social and Behavioural Sciencies EJSBS, XVII, 2029-2054.

Romero, S. (2009). El proyecto vital y profesional. En En L.M. Sobrado y A. Cortés (coords.), Orientación Profesional. Nuevos escenarios y perspectivas (pp.119-142). Madrid: Editorial Biblioteca Nueva.

Romero, S., Seco, M. y Lugo, M. (2015). Orientar desde el Ser: algunas aportaciones del programa Orient@cual para la elaboración del proyecto profesional y Vital de alumnado de PCPI y CFGM. Revista Electrónica Interuniversitaria de Formación del Profesorado, 18(2), 75-89.

Sánchez, C., y Cerezo, F. (2011). Estatus social de los sujetos implicados en bullying. Elementos para la mejora de la convivencia en el aula. REOP, 22(2), 137-149.

Sandoval, M., Simón, C. y Echeita, G. (2019). Educación inclusiva y atención a la diversidad desde la orientación educativa. Madrid: Síntesis.

Santos Guerra, M. A. (2009). El arte de envejecer en la enseñanza. Tendencias Pedagógicas, 14, 365-374.

Segura, M. y Arcas, M. (2003). Educar las emociones y los sentimientos. Introducción práctica al complejo mundo de los sentimientos. Madrid: Narcea.

Sepúlveda, C., Brunaud, V. y Carreño, C. (2016). Justicia Social en la Escuela: Representaciones de Estudiantes de Educación Secundaria y Desafíos para la Formación del Profesorado. Revista Internacional de Educación para la Justicia Social, 5(2), 109-129.

Silva, I., Diniz, J. y Zeichner, K. (Eds). (2017). Justicia social. La dimensión olvidada de la formación docente. Santiago de Chile: Mutante Editores.

Tur, A., Mestre, V., Samper, P. y Malonda, E. (2012). Crianza y agresividad de los menores. Psicothema, 24(2), 284-288.

UNESCO (2015a). Education 2030 Incheon Declaration and Frameworkfor Action: Towards inclusive and equitable quality education and lifelong learning for all. Paris: UNESCO Publishing.

. (2015b). Rethinking Education: Towards a global common good? Paris: UNESCO Publishing.

(2016). Education for people and planet: creating sustainable futures for all. París: UNESCO Publishing.

(2017). Education Transforms Lives. UNESCO Publishing.

(2019). Futures of Education. Learning to become. UNESCO Publishing.

Uribe, P. A. (2018). Percepción de los estudiantes de educación inicial frente al desarrollo de experiencias formativas en modalidad A+S. Revista Electrónica de Investigación Educativa, 20(4), 110-122.

Vaillant, D. (2009). Formación de profesores de Educación Secundaria: realidades y discursos. Revista de Educación, 350, 105-122.

Wasner, V. (2019). Collaborative inquiry into service learning: ethical practice through a Pedagogy of CARE. Journal of Research in International Education, 18(2), 224.

Zhoc, K. C., Chung, T. S. \& King, R. B. (2018). Emotional intelligence (EI) and self-directed learning: Examining their relation and contribution to better student learning outcomes in higher education. British Educational Research Journal, 44(6), 982-1004. 
